\title{
Optimal Adjuvant Treatment for Curatively Resected Thoracic Esophageal Squamous Cell Carcinoma: A Radiotherapy Perspective
}

Kyung Hwan Kim, MD'1
Jee Suk Chang, MD'1
Ji Hye Cha, MD
Ik Jae Lee, MD'
Dae Joon Kim, MD'
Byoung Chul Cho, MD³
Kyung Ran Park, MD
Chang Geol Lee, MD, PhD'

Departments of ${ }^{1}$ Radiation Oncology and ${ }^{2}$ Thoracic and Cardiovascular Surgery, ${ }^{3}$ Devision of Medical Oncology, Department of Internal Medicine, Yonsei Cancer Center, Yonsei University College of Medicine, Seoul, ${ }^{4}$ Department of Radiation Oncology, Ewha Womans University Medical Center, Seoul, Korea
Correspondence: Chang Geol Lee, MD, PhD Department of Radiation Oncology, Yonsei Cancer Center, Yonsei University College of Medicine, 50-1 Yonsei-ro, Seodaemun-gu, Seoul 03722, Korea Tel: $82-2-2228-8114$

Fax: 82-2-312-9033

E-mail: cglee1023@yuhs.ac

\section{Purpose}

The purpose of this study was to evaluate the benefits of adjuvant treatment for curatively resected thoracic esophageal squamous cell carcinoma (ESCC) and determine the optimal adjuvant treatments.

\begin{abstract}
Materials and Methods
One hundred ninety-five patients who underwent a curative resection for thoracic ESCC between 1994 and 2014 were reviewed retrospectively. Postoperatively, the patients received no adjuvant treatment (no-adjuvant group, $n=68$ ), adjuvant chemotherapy (AC group, $n=62$ ), radiotherapy (RT group, $n=41$ ), or chemoradiotherapy (CRT group, $n=24$ ). Chemotherapy comprised cisplatin and 5-fluorouracil administration every 3 weeks. The median RT dose was 45.0 Gy (range, 34.8 to 59.4 Gy). The overall survival (OS), diseasefree survival (DFS), locoregional recurrence (LRR), and distant metastasis (DM) rates were estimated.
\end{abstract}

\section{Results}

At a median follow-up duration of 42.2 months (range, 6.3 to 215.2 months), the 5-year OS and DFS were $37.6 \%$ and $31.4 \%$, respectively. After adjusting for other clinicopathologic variables, the AC and CRT groups had a significantly better OS and DFS compared to the no-adjuvant group $(p<0.05)$. The LRR rate was significantly lower in the RT and CRT groups than in the no-adjuvant group ( $p<0.05$ ), whereas no significant difference was observed in the AC group. In the no-adjuvant and AC groups, $25 \%$ of patients received high-dose salvage RT due to LRR. The DM rates were similar. The anastomotic stenosis and leakage were similar in the treatment groups.

\section{Conclusion}

Adjuvant treatment might prolong survival after an ESCC resection, and RT contributes to a reduction of the LRR. Overall, the risks and benefits should be weighed properly when selecting the optimal adjuvant treatment.

\section{Introduction}

Esophageal cancer is a fatal disease that causes 400,000 deaths per year worldwide [1]. The current guidelines recommend surgery as the mainstay of treatment for esophageal cancer [2,3]. Although neoadjuvant chemoradiotherapy (CRT) is the treatment of choice for locally advanced

\section{Key words}

Esophageal neoplasms, Squamous cell carcinoma, Drug therapy, Radiotherapy esophageal cancer [4], upfront surgery has been performed for several decades. Currently, some patients continue to undergo upfront surgery for a variety of reasons. On the other hand, radical esophagectomy without adjuvant treatment has been associated with suboptimal disease control rates [5]. A few randomized trials have been conducted to evaluate the benefits of adjuvant chemotherapy (AC) and postoperative radiotherapy (RT) [6,7]. Although these trials 
failed to demonstrate a survival benefit of adjuvant treatment, these treatments reduced the recurrence rates significantly. In addition, certain subgroups of patients, such as node-positive patients, who received adjuvant treatment showed significantly higher survival rates $[8,9]$. Recently, some researchers suggested that a combination of chemotherapy and RT might reduce the recurrence rate and improve survival [10].

Unfortunately, there is no clear consensus regarding whether adjuvant treatment is required after a complete resection and what this ideal adjuvant treatment might be. At the authors' institution, the practice has changed from upfront surgery to neoadjuvant treatment followed by surgery in response to the accumulating evidence supporting the benefits of neoadjuvant treatment. On the other hand, some patients without adjacent organ invasion or equivocal lymph node involvement undergo upfront surgery, either with or without adjuvant treatment. Patients with an esophageal squamous cell carcinoma (ESCC) who underwent upfront surgery were reviewed retrospectively to determine the effects of adjuvant treatment and the optimal adjuvant treatment.

\section{Materials and Methods}

\section{Patients}

This study identified 330 patients who underwent a frontline radical resection for nonmetastatic thoracic ESCC between April 1994 and October 2014. None of the patients had received preoperative RT or chemotherapy. Patients with pathological stage T1N0 ( $\mathrm{n}=102)$, a history of other cancer ( $\mathrm{n}=19)$, and / or death within 2 months postoperatively $(\mathrm{n}=14)$ were excluded, yielding a total of 195 patients for analysis.

The preoperative evaluation included a physical examination, laboratory blood tests, esophagogastroduodenoscopy with biopsy, endoscopic ultrasonography, chest computed tomography (CT), abdominopelvic $\mathrm{CT}$, and positron emission tomography scanning.

\section{Surgery}

Details of the surgical resection are described elsewhere $[11,12]$. Briefly, patients with middle and lower esophageal cancers underwent a transthoracic esophagectomy with a two-field lymph node dissection and cervical esophagogastrostomy, using the whole stomach as a conduit (McKeown esophagectomy). Gastric pull-up was performed through the substernal route. A three-field lymph node dissection was performed on the patients with upper esophageal cancer. Beginning in 2006, a robot-assisted thoracoscopic esophagectomy protocol was adopted and the results have been reported [13]. Pathology staging was determined according to the seventh edition of the American Joint Committee on Cancer staging system.

\section{Adjuvant treatment}

Adjuvant treatment was administered 4-6 weeks after surgery in accordance with the pathology risk factors, physicians' preference, patients' performance status, and age. In general, the indications for adjuvant RT included a positive resection margin, locally advanced disease (T3-4), and multiple positive lymph nodes. AC was administered commonly to node-positive patients. Sixty-eight patients received no adjuvant treatment (no-adjuvant group); 62 patients received AC without RT (AC group); 41 patients received RT without chemotherapy (RT group); and 24 patients received both RT and AC (CRT group).

The median RT dose was 45 Gy (range, 34.8 to 59.4 Gy), which was delivered in daily fraction doses of $1.8 \mathrm{~Gy}$. One patient did not complete the planned treatment of $45 \mathrm{~Gy}$ and refused further treatment after receiving $34.8 \mathrm{~Gy}$. The RT field included the tumor bed, anastomosis site, and lymph node drainage areas. Although the supraclavicular fossa was not irradiated routinely, it was performed on the patients with upper esophageal cancer or involvement of the supraclavicular lymph nodes.

The chemotherapy regimen comprised the intravenous infusion of cisplatin and 5-fluorouracil every 3 weeks for 4-6 cycles. For the patients in the CRT group, RT was initiated 1 week after the completion of two cycles of chemotherapy in a sequential manner. Chemotherapy was re-initiated after the completion of RT.

\section{Follow-up evaluation}

The patients were followed up every 3 months after the esophagectomy for the first year, every 6 months for the next 2 years, and annually thereafter. Abdominopelvic CT was performed every 6-12 months and an esophagogastroduodenoscopy was performed annually. The incidence of postoperative complications, such as anastomotic stenosis and leakage, were evaluated. Endoscopic balloon dilatation was performed when symptomatic anastomotic stenosis was evident and prevented the passage of the gastroduodenoscope. A biopsy was performed if a malignant anastomotic stricture was suspected.

Recurrences at the anastomotic site, tumor bed, and regional lymph node area were considered locoregional, 
Table 1. Characteristics of the patients in the four treatment groups

\begin{tabular}{|c|c|c|c|c|c|}
\hline Characteristic & $\begin{array}{l}\text { No-adjuvant } \\
\quad(n=68)\end{array}$ & $\begin{array}{c}\mathrm{AC} \\
(\mathrm{n}=62)\end{array}$ & $\begin{array}{c}\mathrm{RT} \\
(\mathrm{n}=41)\end{array}$ & $\begin{array}{c}\text { CRT } \\
(n=24)\end{array}$ & p-value \\
\hline \multicolumn{6}{|l|}{ Age (yr) } \\
\hline$\leq 60$ & $20(29.4)$ & $32(51.6)$ & $22(53.7)$ & $15(62.5)$ & 0.008 \\
\hline$>60$ & $48(70.6)$ & $30(48.4)$ & $19(46.3)$ & $9(37.5)$ & \\
\hline \multicolumn{6}{|l|}{ Sex } \\
\hline Male & $58(85.3)$ & $57(91.9)$ & $40(97.6)$ & $21(87.5)$ & $0.166^{\mathrm{a})}$ \\
\hline Female & $10(14.7)$ & $5(8.1)$ & $1(2.4)$ & $3(12.5)$ & \\
\hline \multicolumn{6}{|l|}{ ECOG performance status } \\
\hline 0 & $52(76.5)$ & $56(90.3)$ & $26(63.4)$ & $17(70.8)$ & 0.117 \\
\hline 1 & $16(23.5)$ & $6(19.7)$ & $15(36.6)$ & $7(29.2)$ & \\
\hline \multicolumn{6}{|l|}{ Location } \\
\hline Upper to mid thoracic & $32(47.1)$ & $21(33.9)$ & $17(41.5)$ & $7(29.2)$ & 0.310 \\
\hline Lower thoracic to EGJ & $36(52.9)$ & $41(66.1)$ & $24(58.5)$ & $17(70.8)$ & \\
\hline \multicolumn{6}{|l|}{ Histologic grade } \\
\hline Grade 1 or unknown & $9(13.2)$ & $14(22.6)$ & $12(29.3)$ & $3(12.5)$ & 0.150 \\
\hline Grade $2-3$ & $59(86.8)$ & $48(77.4)$ & $29(70.7)$ & $21(87.5)$ & \\
\hline \multicolumn{6}{|l|}{ Tumor length (cm) } \\
\hline$\leq 5$ & $58(85.3)$ & $47(75.8)$ & $29(70.7)$ & $17(70.8)$ & 0.246 \\
\hline$>5$ & $10(14.7)$ & $15(24.2)$ & $12(29.3)$ & $7(29.2)$ & \\
\hline \multicolumn{6}{|l|}{$\mathrm{T}$ classification } \\
\hline $\mathrm{T} 1$ & $13(19.1)$ & $16(25.8)$ & $1(2.4)$ & $7(29.2)$ & $0.003^{\mathrm{a})}$ \\
\hline $\mathrm{T} 2$ & $28(41.2)$ & $14(22.6)$ & $9(22.0)$ & $5(20.8)$ & \\
\hline T3 & $26(38.2)$ & $32(51.6)$ & $31(75.6)$ & $12(50.0)$ & \\
\hline $\mathrm{T} 4$ & $1(1.5)$ & 0 & 0 & 0 & \\
\hline \multicolumn{6}{|l|}{$\mathrm{N}$ classification } \\
\hline No & $34(50.0)$ & $7(11.3)$ & $17(41.5)$ & $3(12.5)$ & $<0.001^{\text {a) }}$ \\
\hline N1 & $24(35.3)$ & $31(50.0)$ & $16(39.0)$ & $15(62.5)$ & \\
\hline N2 & $8(11.8)$ & $17(27.4)$ & $6(14.6)$ & $5(20.8)$ & \\
\hline N3 & $2(2.9)$ & $7(11.3)$ & $2(4.9)$ & $1(4.2)$ & \\
\hline \multicolumn{6}{|l|}{ Lymphovascular invasion } \\
\hline No & $56(82.4)$ & $43(69.4)$ & $33(80.5)$ & $15(62.5)$ & 0.126 \\
\hline Yes & $12(17.6)$ & $19(30.6)$ & $8(19.5)$ & $9(37.5)$ & \\
\hline \multicolumn{6}{|l|}{ Perineural invasion } \\
\hline No & $64(94.1)$ & $55(88.7)$ & $37(90.2)$ & $23(95.8)$ & $0.618^{\mathrm{a})}$ \\
\hline Yes & $4(5.9)$ & $7(11.3)$ & $4(9.8)$ & $1(4.2)$ & \\
\hline \multicolumn{6}{|l|}{ Resection margin } \\
\hline R0 & $66(97.1)$ & $58(93.5)$ & $38(92.7)$ & $24(100)$ & $0.506^{\mathrm{a})}$ \\
\hline $\mathrm{R} 1$ & $2(2.9)$ & $4(6.5)$ & $3(7.3)$ & 0 & \\
\hline \multicolumn{6}{|l|}{ Extracapsular extension } \\
\hline No & $61(89.7)$ & $46(74.2)$ & $30(73.2)$ & $20(83.3)$ & 0.080 \\
\hline Yes & $7(10.3)$ & $16(25.8)$ & $11(26.8)$ & $4(16.7)$ & \\
\hline \multicolumn{6}{|l|}{ Treated period } \\
\hline 1994 to 2002 & $32(47.1)$ & $26(41.9)$ & $30(73.2)$ & $11(45.8)$ & 0.013 \\
\hline 2003 to 2014 & $36(52.9)$ & $36(58.1)$ & $11(26.8)$ & $13(54.2)$ & \\
\hline
\end{tabular}

AC, adjuvant chemotherapy; RT, radiotherapy; CRT, chemoradiotherapy; ECOG, Eastern Cooperative Oncology Group; EGJ, esophagogastric junction. ${ }^{\text {a) }}$ Fisher exact test. 
A

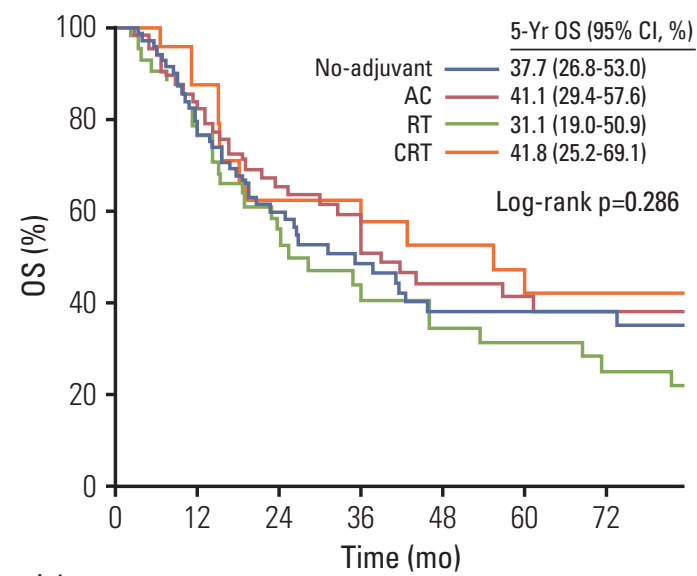

No. at risk

$\begin{array}{rrrrrrrr}\text { No-adjuvant } & 68 & 53 & 36 & 23 & 16 & 15 & 13 \\ \text { AC } & 62 & 51 & 36 & 25 & 16 & 12 & 11 \\ \text { RT } & 41 & 32 & 20 & 14 & 11 & 10 & 8 \\ \text { CRT } & 24 & 21 & 13 & 12 & 10 & 8 & 8\end{array}$

C

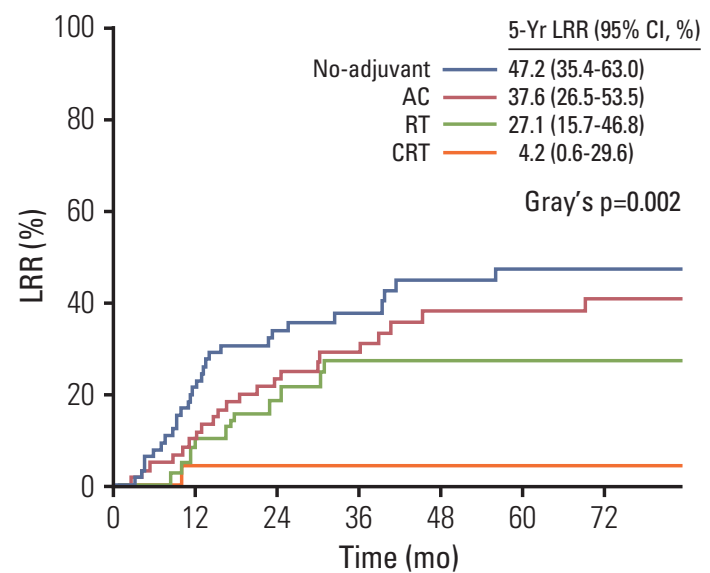

No. at risk

$\begin{array}{llllllll}\text { No-adjuvant } & 68 & 44 & 30 & 19 & 13 & 12 & 9\end{array}$

$\begin{array}{rrrrrrrr}\text { AC } & 62 & 48 & 32 & 21 & 14 & 10 & 9 \\ \text { RT } & 41 & 30 & 19 & 10 & 10 & 9 & 8 \\ \text { CRT } & 24 & 20 & 13 & 12 & 10 & 8 & 8\end{array}$

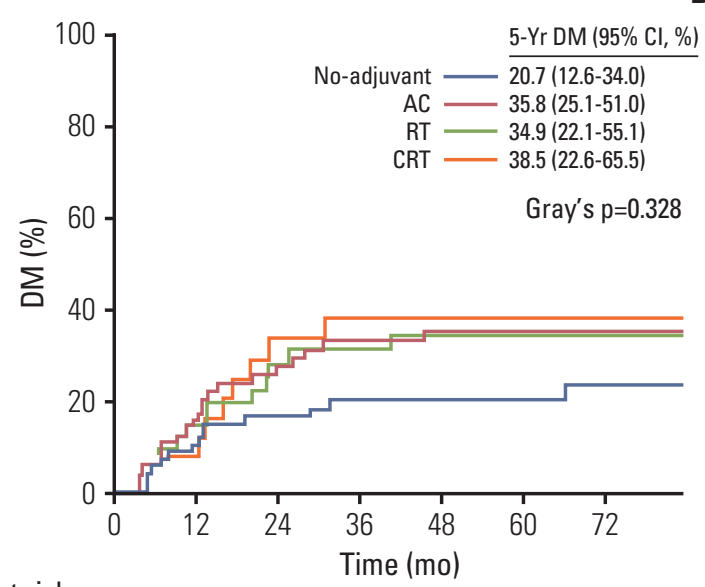

No. at risk

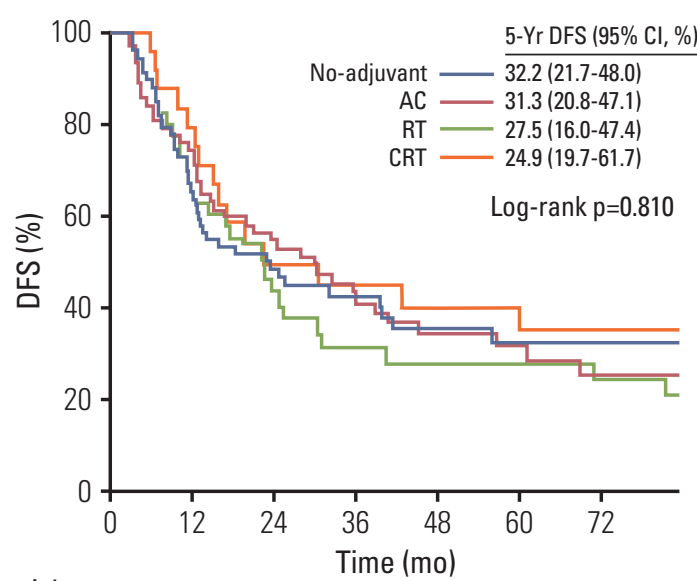

No. at risk

$\begin{array}{rrrrrrrr}\text { No-adjuvant } & 68 & 43 & 28 & 18 & 13 & 11 & 9 \\ \text { AC } & 62 & 46 & 31 & 20 & 14 & 10 & 8 \\ \text { RT } & 41 & 27 & 15 & 9 & 8 & 8 & 7 \\ \text { CRT } & 24 & 19 & 11 & 10 & 8 & 7 & 7\end{array}$

$\begin{array}{rrrrrrrr}\text { No-adjuvant } & 68 & 51 & 34 & 21 & 14 & 14 & 10 \\ \text { AC } & 62 & 47 & 34 & 23 & 15 & 11 & 10 \\ \text { RT } & 41 & 29 & 15 & 12 & 8 & 8 & 7 \\ \text { CRT } & 24 & 20 & 11 & 10 & 8 & 7 & 7\end{array}$

Fig. 1. Estimated overall survival (OS) (A), disease-free survival (DFS) (B), locoregional recurrence (LRR) (C), and distant metastasis (DM) rates (D) of the patients who received no adjuvant treatment, adjuvant chemotherapy (AC), postoperative radiotherapy (RT), and postoperative chemoradiotherapy (CRT). CI, confidence interval. 
whereas recurrences elsewhere were considered distant.

\section{Statistical analysis}

A Pearson's chi-square test or Fisher exact test was performed where appropriate to compare the characteristics of the different patient groups. The overall survival (OS), disease-free survival (DFS), locoregional recurrence (LRR), and distant metastasis $(\mathrm{DM})$ rates were calculated from the date of surgery to the date of death from any cause, any recurrence or death, LRR, and distant metastasis, respectively. The survival endpoints were analyzed using the Kaplan-Meier method with a log-rank test for comparisons of the three treatment groups. The LRR and DM rates were estimated using the cumulative incidence method and Gray's test [14]. To adjust for the effects of other variables on the treatment outcome of the different adjuvant treatments, multivariable models were generated to include the factors with $\mathrm{p}$-values of $<0.10$ in univariate analyses. Cox proportional hazards regression was used to conduct univariate and multivariate analyses of the OS and DFS. For LRR and DM, univariate and multivariate models were generated by Fine and Gray regression analysis [15]. All tests were two-sided and considered significant at a $p$-value $<0.05$. Statistical analyses were performed using IBM SPSS ver. 20.0 (IBM Corp., Armonk, NY) and R software ver. 3.2.2 (R Development Core Team, Vienna, Austria).

\section{Results}

\section{Patient and treatment characteristics}

Table 1 lists the characteristics of the patients in the noadjuvant, AC, RT, and CRT groups. The no-adjuvant group included a larger number of elderly patients, whereas the AC, RT, and CRT groups included more locally advanced patients. The majority of patients who received adjuvant treatment had lymph node involvement and adventitial invasion. Fewer patients were treated with RT alone in the more recent period. The tumor location, histology grade, tumor length, and other histologic risk factors were similar in the three groups. Most patients underwent a McKeown esophagectomy $(n=159)$, whereas others underwent an IvorLewis $(n=21)$ or transhiatal esophagectomy $(n=15)$.

A median of four cycles (range, 1 to 6 ) of chemotherapy were administered. Nine patients (eight in the AC group and one in the CRT group) did not complete the planned chemotherapy due to poor performance $(n=6)$ and refusal $(n=3)$. One patient failed to complete the full course of RT, and 22 patients had a treatment break during RT.

\section{Survival rates according to different adjuvant treatments}

The median follow-up duration was 42.2 months (range, 6.3 to 215.2 months). The median OS and DFS durations were 36.0 months (95\% confidence interval [CI], 27.5 to 44.5 ) and 23.7 months (95\% CI, 16.5 to 30.9), respectively. The 5-year OS and DFS rates were $37.6 \%$ (95\% CI, 30.7 to 46.0 ) and $31.4 \%$ (95\% CI, 24.9 to 39.5), respectively. The OS and DFS were similar in the different treatment groups (Fig. 1A and B). In particular, sex, $\mathrm{T}$ classification, $\mathrm{N}$ classification, lymphovascular invasion, resection margin, and treated period were significant factors associated with both DFS and OS (Table 2). After adjusting for these significant clinicopathological factors, the AC (adjusted hazard ratio [aHR], 0.47; 95\% CI, 0.28 to 0.77 ) and CRT groups (aHR, $0.52 ; 95 \% \mathrm{CI}, 0.29$ to 0.96 ) exhibited a significantly lower risk of death relative to the no-adjuvant group. In addition, the AC (aHR, 0.56; 95\% CI, 0.35 to 0.90 ), RT (aHR, $0.58 ; 95 \% \mathrm{CI}, 0.35$ to 0.98 ), and CRT groups (aHR, $0.51 ; 95 \% \mathrm{CI}, 0.28$ to 0.91 ) exhibited a significantly lower risk of recurrence or death compared to the no-adjuvant group.

\section{Patterns of initial recurrence and recurrence rates}

Table 3 provided the data concerning recurrences. LRR was the dominant pattern of recurrence in the no-adjuvant group, whereas DM was dominant in the RT and CRT groups. The rates of LRR and DM were similar in the AC group. The 5-year LRR rate was lowest in the CRT group (Fig. 1C). Compared to the no-adjuvant group, the RT and CRT groups had a significantly lower risk of LRR, whereas the AC group did not. After adjusting for the significant factors, the RT (aHR, $0.26 ; 95 \% \mathrm{CI}, 0.13$ to 0.54 ) and CRT groups (aHR, $0.05 ; 95 \%$ CI, 0.01 to 0.39 ) exhibited a significantly lower risk of LRR (Table 4). The 5-year DM incidence was similar in the treatment groups ( $\mathrm{p}=0.186)$ (Fig. 1D), even after the adjustment (Table 4).

\section{Application of RT after LRR}

A total of 52 patients who did not receive postoperative RT experienced LRR. Among these patients, 40 underwent salvage treatment for the recurrent lesions; locoregional RT was delivered to 33 patients, including 18 in the no-adjuvant group and 15 in the AC group. A median dose of 59.4 Gy (range, 14.4 to $63.0 \mathrm{~Gy}$ ) was delivered to these patients. After salvage RT, 18 patients exhibited a partial response or stable disease, nine progressed, and six were nonevaluable according to the Response Evaluation Criteria in Solid Tumors ver. 1.1 [16]. The median survival duration after salvage RT was 


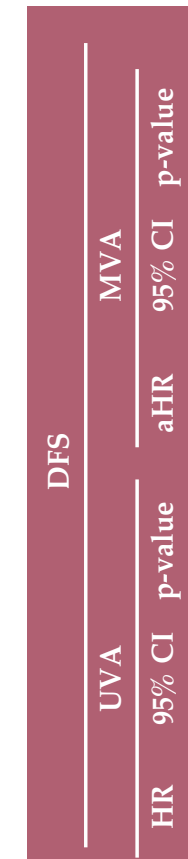

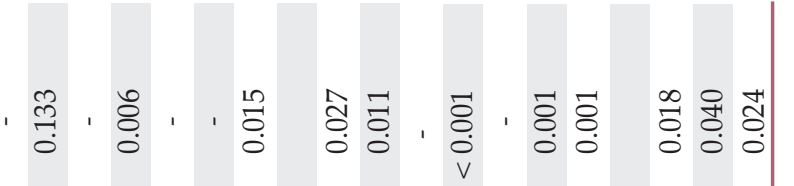

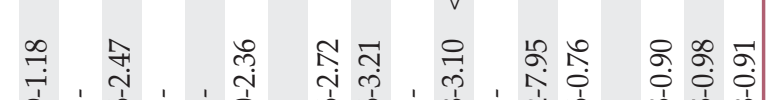

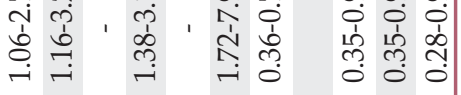

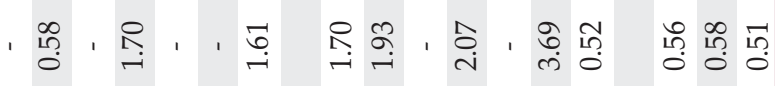

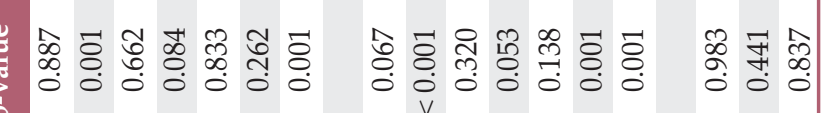

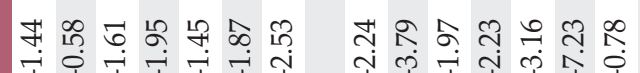

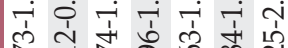

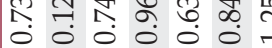

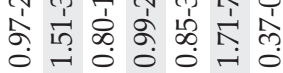

당 तิ

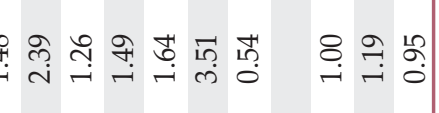

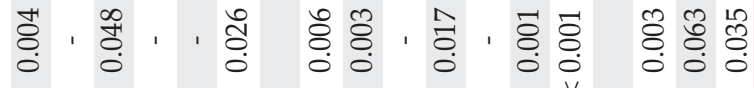

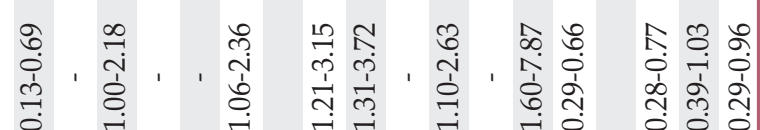

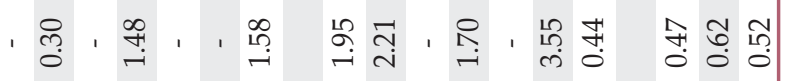

里

\&

光

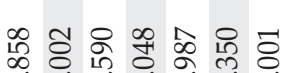

픔

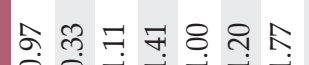

oू.

న้

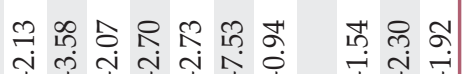

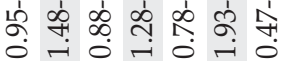
过尔 O H H

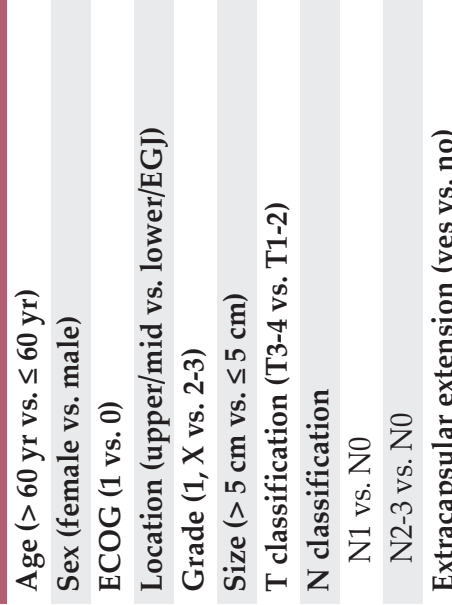

국

$\stackrel{\infty}{\circ} \stackrel{9}{\rightarrow} \stackrel{9}{=}$

总

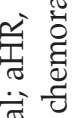

焉

क्षे

氙灾

䆑

플

政

के

芩:

.̦

है

当

还蓄

is

สี

营

帘

过

3 要 
Table 3. Patterns of initial recurrence

\begin{tabular}{lcccc} 
Event & $\begin{array}{c}\text { No-adjuvant } \\
(\mathbf{n}=\mathbf{6 8})\end{array}$ & $\begin{array}{c}\text { AC } \\
(\mathbf{n}=62)\end{array}$ & $\begin{array}{c}\text { RT } \\
(\mathbf{n}=\mathbf{4 1})\end{array}$ \\
Locoregional recurrence & $28(41.2)$ & $24(35.3)$ & $10(24.4)$ & $1(4.2)$ \\
$\quad$ Local only & $6(8.8)$ & $2(2.9)$ & $2(4.9)$ & 0 \\
$\quad$ Regional only & $19(27.9)$ & $17(25.0)$ & $8(19.5)$ & $1(4.2)$ \\
$\quad$ Local and regional & $3(4.4)$ & $5(7.4)$ & 0 & 0 \\
Distant recurrence & $14(20.6)$ & $22(35.5)$ & $13(31.7)$ & $9(37.5)$ \\
Concurrent locoregional and distant recurrence & $8(11.8)$ & $11(16.2)$ & $2(4.9)$ & 0 \\
\hline
\end{tabular}

Values are presented as number (\%). AC, adjuvant chemotherapy; RT, radiotherapy; CRT, chemoradiotherapy.

10.1 months and was 15.2 months for those who did not progress after salvage RT.

\section{Postoperative complications}

The overall incidence of anastomotic stenosis requiring intervention (e.g., endoscopic balloon dilatation) was $29.2 \%$, and was similar in the no-adjuvant, $\mathrm{AC}, \mathrm{RT}$, and CRT groups ( $29.4 \%$ vs. $30.6 \%$ vs. $26.8 \%$ vs. $29.2 \%$, p=0.991). Anastomotic leakage was an uncommon event with an overall incidence of $3.6 \%$; no significant differences were observed among the no-adjuvant, AC, RT, and CRT groups ( $1.5 \%$ vs. $4.8 \%$ vs. $4.9 \%$ vs. $4.2 \%, \mathrm{p}=0.651)$. None of the patients who received RT suffered from severe radiation-induced gastritis.

\section{Discussion}

In the present study, patients with resected ESCC who received $A C$ and postoperative CRT showed significantly higher survival rates than those who did not receive adjuvant treatment. In terms of LRR, those who received adjuvant treatments that included RT experienced significantly less LRRs than those who did not receive adjuvant treatment. Currently, patients with locally advanced ESCC are treated preferably with preoperative CRT followed by a resection, rather than upfront surgery [4]. On the other hand, a certain portion of patients may undergo upfront surgery because of the patients' or physicians' preferences, an inability to receive preoperative chemotherapy or RT, and/or minimally advanced-stage disease. In such cases, the current guidelines do not suggest further treatment after a R0 resection for ESCC because of a lack of randomized evidence of improved survival after adjuvant treatment [2,3].

Several randomized trials that compared surgery with or without postoperative RT demonstrated a lack of a survival benefit [7,17-19]. The most recently conducted randomized trial, however, showed that stage III patients gained a significant survival benefit from postoperative RT [7]. In Japan, a randomized controlled trial that compared surgery with and without AC failed to show a significant OS benefit [6]. In contrast, several retrospective studies have shown higher survival rates in patients with positive lymph nodes or stage III disease who received postoperative RT or AC compared to the no-adjuvant treatment [8,20-23]. Similarly, in the present study, the AC and CRT group showed a significantly lower risk of death compared to the no-adjuvant group even after adjusting for other clinicopathologic variables. Most patients in this study had either lymph node involvement or local invasion beyond the muscularis propria. For patients with less advanced disease after a resection, adjuvant treatment might not yield a demonstrably significant survival benefit.

No randomized controlled trials have been conducted to compare the treatment outcomes of different adjuvant treatments. Chen et al. [24] reviewed retrospectively 366 patients with mid-thoracic ESCC who underwent an esophagectomy followed by no-adjuvant treatment, AC, or RT. Although they did not compare the survival, they found that patients who received RT had a significantly lower incidence of regional recurrence. On the other hand, a remaining concern regarding postoperative $\mathrm{RT}$ is that it cannot prevent recurrences beyond the radiation field; therefore, distant metastases comprise the major failure sites [21]. Despite the limited data, a recent report on patients with node-positive ESCC showed that patients receiving both chemotherapy and postoperative RT had lower recurrences rates, including distant metastases, and superior OS compared to those receiving postoperative RT only [10]. According to the data in the present study, the CRT group, not the RT group, showed significantly higher OS than the no-adjuvant group. In addition, the LRR risk reduction rate was more prominent in the CRT group than in the RT group. No difference in DM was observed with the addition of chemotherapy. The most plausible explanation for these findings appears to be an imbal- 


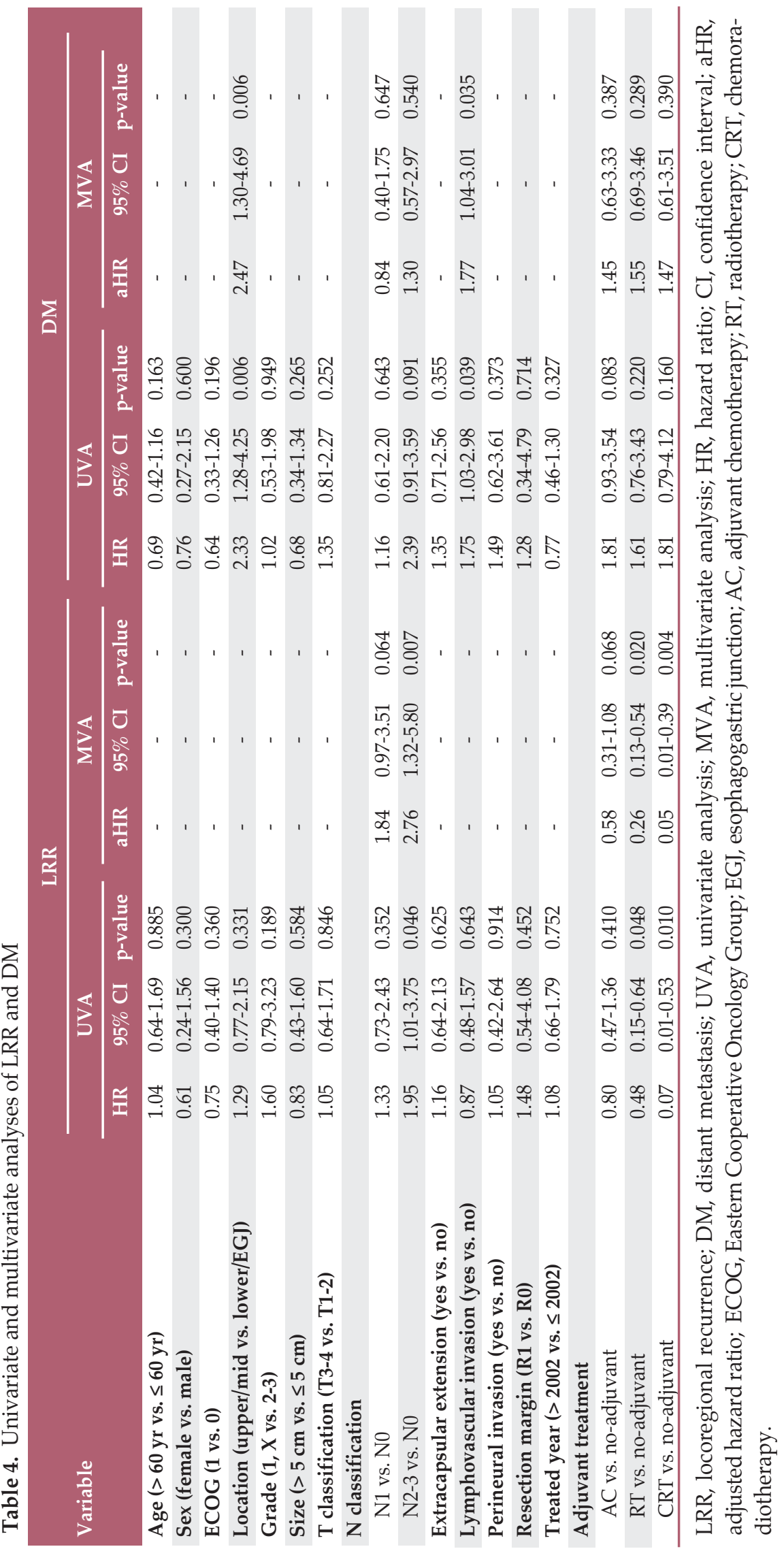


ance in the tumor characteristics among the different treatment groups. Patients who received chemotherapy were more likely to have the risk factors for distant metastasis, such as lymph node involvement and lymphovascular invasion compared to those who did not receive AC.

When deciding the optimal adjuvant treatment, the risks and benefits of the treatment should be measured. This study suggests that both AC and CRT might effectively increase the OS; therefore, AC would be a better option because RT could be spared. On the other hand, 25\% (33 of 130) of the patients who did not receive postoperative RT ultimately received locoregional RT because of LRR; these patients comprised 63\% (33 of 52) of those who developed a LRR. A median dose of $60 \mathrm{~Gy}$ was delivered to these recurrent patients, which is considered a definitive dose for gross tumor eradication. This suggests that $75 \%$ of patients could have been spared unnecessary RT. More than half of the patients responded to salvage RT, leading to a median survival of 15 months after recurrence. A previous phase II trial reported a median survival of 35 months after salvage CRT [25]. These salvage rates, however, were consequent to highdose irradiation to the mediastinum. In particular, the dose delivered in a recurrent setting is higher than that administered to patients receiving postoperative RT (45-50 Gy). In patients subjected to thoracic surgery, the actual effects of RT on the surrounding normal organs might be more prominent than the anticipated effects (based on nonoperated patients), and a higher dose might cause fatal damage to the surrounding organs. Therefore, patients at a higher risk of LRR might benefit most from RT because it could reduce the need for subsequent high-dose RT.

One of the greatest concerns associated with postoperative RT after esophagectomy is the risk of anastomotic leakage and stenosis. A randomized trial reported an increased incidence of anastomotic stenosis after postoperative RT [17]. In contrast, a more recent randomized trial observed no significant increase in the incidence of anastomotic stenosis after postoperative RT [7]. These results also support the finding that postoperative RT does not increase significantly the incidence of anastomotic stenosis or leakage after esophagectomy. In addition, severe gastritis was not observed after RT, mainly because of the surgical technique that positioned the stomach conduit through the substernal route. Accordingly, the stomach remained outside of the RT field.

Several limitations stemmed from the retrospective nature of this study. Adjuvant treatment selection was nonrandomized, and there were significant differences in the patient characteristics among the study groups. An attempt was made to adjust for these imbalances in characteristics through multivariate analysis. In addition, data on the complications or patients' quality of life, which are also important endpoints, were not collected prospectively. Finally, the patient number in each treatment group was relatively small, and the statistical power was low.

\section{Conclusion}

CRT and AC might be considered relevant options for adjuvant treatment in patients with a resected ESCC. Assuming that the patient can tolerate both chemotherapy and RT, CRT may be an optimal choice because it could spare the patients from later high-dose irradiation for LRR without causing an increase in the long-term postsurgical complications. On the other hand, this study cannot draw conclusions regarding the most beneficial adjuvant treatment because of the nonrandomized study design and the afore-mentioned limitations. Additional well-designed prospective randomized trials will be needed to confirm these results.

\section{Conflicts of Interest}

Conflict of interest relevant to this article was not reported.

\section{References}

1. Torre LA, Bray F, Siegel RL, Ferlay J, Lortet-Tieulent J, Jemal A. Global cancer statistics, 2012. CA Cancer J Clin. 2015;65:87108.

2. Ajani JA, D'Amico TA, Almhanna K, Bentrem DJ, Besh S, Chao J, et al. Esophageal and esophagogastric junction cancers, version 1.2015. J Natl Compr Canc Netw. 2015;13:194-227.

3. Stahl M, Mariette C, Haustermans K, Cervantes A, Arnold D;
ESMO Guidelines Working Group. Oesophageal cancer: ESMO Clinical Practice Guidelines for diagnosis, treatment and follow-up. Ann Oncol. 2013;24 Suppl 6:vi51-6.

4. van Hagen P, Hulshof MC, van Lanschot JJ, Steyerberg EW, van Berge Henegouwen MI, Wijnhoven BP, et al. Preoperative chemoradiotherapy for esophageal or junctional cancer. $\mathrm{N}$ Engl J Med. 2012;366:2074-84. 
5. Nakagawa S, Kanda T, Kosugi S, Ohashi M, Suzuki T, Hatakeyama K. Recurrence pattern of squamous cell carcinoma of the thoracic esophagus after extended radical esophagectomy with three-field lymphadenectomy. J Am Coll Surg. 2004;198:205-11.

6. Ando N, Iizuka T, Ide H, Ishida K, Shinoda M, Nishimaki T, et al. Surgery plus chemotherapy compared with surgery alone for localized squamous cell carcinoma of the thoracic esophagus: a Japan Clinical Oncology Group Study: JCOG 9204. J Clin Oncol. 2003;21:4592-6.

7. Xiao ZF, Yang ZY, Liang J, Miao YJ, Wang M, Yin WB, et al. Value of radiotherapy after radical surgery for esophageal carcinoma: a report of 495 patients. Ann Thorac Surg. 2003;75:3316.

8. Lyu X, Huang J, Mao Y, Liu Y, Feng Q, Shao K, et al. Adjuvant chemotherapy after esophagectomy: is there a role in the treatment of the lymph node positive thoracic esophageal squamous cell carcinoma? J Surg Oncol. 2014;110:864-8.

9. Xu Y, Liu J, Du X, Sun X, Zheng Y, Chen J, et al. Prognostic impact of postoperative radiation in patients undergoing radical esophagectomy for pathologic lymph node positive esophageal cancer. Radiat Oncol. 2013;8:116.

10. Chen J, Pan J, Liu J, Li J, Zhu K, Zheng X, et al. Postoperative radiation therapy with or without concurrent chemotherapy for node-positive thoracic esophageal squamous cell carcinoma. Int J Radiat Oncol Biol Phys. 2013;86:671-7.

11. Kim DJ, Hyung WJ, Lee CY, Lee JG, Haam SJ, Park IK, et al. Thoracoscopic esophagectomy for esophageal cancer: feasibility and safety of robotic assistance in the prone position. J Thorac Cardiovasc Surg. 2010;139:53-9.

12. Park SY, Lee HS, Jang HJ, Lee JY, Joo J, Zo JI. The role of oneyear endoscopic follow-up for the esophageal remnant and gastric conduit after esophagectomy with gastric reconstruction for esophageal squamous cell carcinoma. Yonsei Med J. 2013;54:381-8.

13. Park SY, Kim DJ, Yu WS, Jung HS. Robot-assisted thoracoscopic esophagectomy with extensive mediastinal lymphadenectomy: experience with 114 consecutive patients with intrathoracic esophageal cancer. Dis Esophagus. 2016;29:32632.

14. Gray RJ. A class of K-sample tests for comparing the cumulative incidence of a competing risk. Ann Stat. 1988;16:1141-54.

15. Fine JP, Gray RJ. A proportional hazards model for the subdistribution of a competing risk. J Am Stat Assoc. 1999;94:496509.

16. Eisenhauer EA, Therasse P, Bogaerts J, Schwartz LH, Sargent
D, Ford R, et al. New response evaluation criteria in solid tumours: revised RECIST guideline (version 1.1). Eur J Cancer. 2009;45:228-47.

17. Zieren HU, Muller JM, Jacobi CA, Pichlmaier H, Muller RP, Staar S. Adjuvant postoperative radiation therapy after curative resection of squamous cell carcinoma of the thoracic esophagus: a prospective randomized study. World J Surg. 1995;19:444-9.

18. Fok M, Sham JS, Choy D, Cheng SW, Wong J. Postoperative radiotherapy for carcinoma of the esophagus: a prospective, randomized controlled study. Surgery. 1993;113:138-47.

19. Teniere P, Hay JM, Fingerhut A, Fagniez PL. Postoperative radiation therapy does not increase survival after curative resection for squamous cell carcinoma of the middle and lower esophagus as shown by a multicenter controlled trial. French University Association for Surgical Research. Surg Gynecol Obstet. 1991;173:123-30.

20. Xiao ZF, Yang ZY, Miao YJ, Wang LH, Yin WB, Gu XZ, et al. Influence of number of metastatic lymph nodes on survival of curative resected thoracic esophageal cancer patients and value of radiotherapy: report of 549 cases. Int J Radiat Oncol Biol Phys. 2005;62:82-90.

21. Chen J, Pan J, Zheng X, Zhu K, Li J, Chen M, et al. Number and location of positive nodes, postoperative radiotherapy, and survival after esophagectomy with three-field lymph node dissection for thoracic esophageal squamous cell carcinoma. Int J Radiat Oncol Biol Phys. 2012;82:475-82.

22. Schreiber D, Rineer J, Vongtama D, Wortham A, Han P, Schwartz D, et al. Impact of postoperative radiation after esophagectomy for esophageal cancer. J Thorac Oncol. 2010;5: 244-50.

23. Zhang J, Zhang YW, Chen ZW, Zhou XY, Lu S, Luo QQ, et al. Adjuvant chemotherapy of cisplatin, 5-fluorouracil and leucovorin for complete resectable esophageal cancer: a casematched cohort study in east China. Dis Esophagus. 2008; 21:207-13.

24. Chen G, Wang Z, Liu XY, Liu FY. Adjuvant radiotherapy after modified Ivor-Lewis esophagectomy: can it prevent lymph node recurrence of the mid-thoracic esophageal carcinoma? Ann Thorac Surg. 2009;87:1697-702.

25. Ma DY, Tan BX, Liu M, Li XF, Zhou YQ, Lu Y. Concurrent three-dimensional conformal radiotherapy and chemotherapy for postoperative recurrence of mediastinal lymph node metastases in patients with esophageal squamous cell carcinoma: a phase 2 single-institution study. Radiat Oncol. 2014; 9:28. 